\section{Independence of mind}

\section{Michael Berry}

\section{Joumey Into Ught: Life and Science of C.V. Raman. By G. Venkataraman. Indian Academy of Sciences: 1988. Pp. 570. Distributed in Britain and the United States by Oxford University Press, $£ 22.50$.}

THE past two years have seen centenaries of the birth of several of India's most brilliant citizens: Nehru, founder of the modern state; Ramanujan, diviner of amazing mathematical formulae; and Raman, the prolific and original physicist whom this book celebrates. Raman's life and work were complex and many-sided, causing great difficulties of judgement and emphasis for any biographer. Venkataraman (who in spite of his name does not share with several other talented Indian scientists the distinction of being a relative of Raman) solves these problems by anchoring his story firmly in the science. He has produced a model of scientific biography, written with respect and affection for its subject but with a clear-eyed perception of his faults, in a relaxed and gently witty style.

Raman's work was on the physics of waves and overwhelmingly centred on optics. He is best known for the effect that bears his name, but that was not discovered until he was 40 years old, when he was already established internationally as an authority on a variety of subtle, classical interference and diffraction phenomena such as the colours of heated metals and layers of bubbles. He had also studied the production of musical tones. An interesting discovery was that Indian drums have skins whose thickness varies radially in such a way that the frequencies of the overtones are integer multiples of the fundamental, so that they sound more harmonious than their Western counterparts, whose skins are radially uniform and generate irrational overtones.

The Raman effect is the scattering of light with a change of frequency (that is, inelastic scattering); it occurs in liquids, solids and isolated molecules. The change in frequency occurs when the light exchanges energy with the internal vibration or rotation states of whatever scatters the light. Compared with the elastic, or Rayleigh, scattering, for which the incident and scattered beams have the same frequency, the Raman effect is weak, which is why it was not discovered earlier. Raman (with Krishnan) found it by deploying simple apparatus with great experimental skill. The discovery was announced in Nature. Venkataraman mentions anecdotal evidence that the paper was first rejected. New evidence confirms this: apparently there were two unfavourable referees, both Fellows of the Royal Society, who provoked in the editor (Sir Richard Gregory) the opinion that any paper inspiring such vehement opposition must have something good in it and so should be published! The paper stimulated a great deal of immediate and continuing interest, not only by demonstrating a fundamental optical effect fully concordant with the (then) new quantum mechanics but also by providing a sensitive probe of the vibrational and (for molecules) rotational structure of matter.

Raman's creative work did not stop

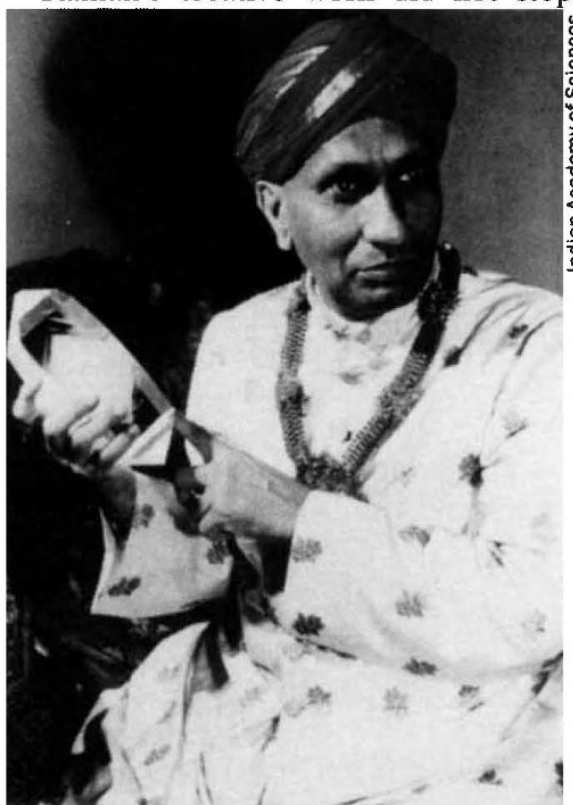

Offering enlightenment - Raman on crystals. with the Raman effect. I was surprised to learn that he discovered or anticipated several phenomena usually regarded as having been found decades later by others. One of these is what is now called 'speckle'. This is the mottled appearance of coherent light scattered by static randomness such as grains of powder on a screen or irregularities on a rough painted wall. Nowadays speckle is a familiar accompaniment of images produced by lasers, but Raman (with Ramachandran) saw it in light from a mercury lamp filtered by a pinhole. He had a complete understanding of the phenomenon, 20 years before lasers were invented.

Another anticipation is of what are now called 'soft modes'. A soft mode in a solid is a vibration whose frequency vanishes as a critical temperature is approached. The vanishing indicates the weakening of the structure, which changes abruptly at the critical temperature. Raman (with Nedungadi), using Raman spectroscopy, observed the decrease in a lattice vibration frequency of quartz and correctly understood its association with a structural phase transition.

A high point of Raman's work after the discovery of the Raman effect was his theory (with Nath) of the diffraction patterns produced by light traversing refractive-index corrugations made by irradiating a liquid with ultrasound. As Venkataraman puts it, in a chapter charmingly titled "Son et lumière",

Raman loved waves, and this problem had light waves as well as sound waves. What more could he ask for? . . . The full-fledged Raman-Nath theory was put to a stringent test only in 1963 . . . [and] is as good as one would want it to be, provided one takes the trouble of carefully solving the equations.

Recently I learned that this theory too is an anticipation: exactly the same equations have been rediscovered in the theory of free-electron lasers.

The failures of great scientists are often as interesting as their successes, and Raman had his share of failures. Perhaps the best known is his controversy with Born about the vibration spectrum of diamond. Because of a persistent mathematical misunderstanding of the dynamics of crystal lattices, he never accepted the fact that the vibration frequencies form a continuum. He predicted a discrete spectrum, on the basis of a 'quasi-molecular' theory of his own, and he carried out experiments that seemed to confirm it. As Venkataraman explains, Raman's theory, although wrong, picked out a particular subset of the vibrational modes, at which the spectrum has singularities that Born did not know about but whose existence was later demonstrated by Van Hove. His experiments had fairly low resolution and so he saw only the singularities, masquerading as sharp lines. In other words,

Raman was locally correct but globally wrong. The part he got right is an important part and reflects his native brilliance. He would not have erred the way he did had he been trained properly in quantum physics.

I long suspected that Raman never properly understood the connection between wave optics and geometrical optics. That suspicion is confirmed by the account here of his ideas about the mirage. By imagining the air above a hot surface as a stack of slabs of slightly different refractive indices, he convinced himself that refraction could never make a downwardsloping ray turn upwards, because a ray once horizontal would remain so. In other words, refraction could never simulate reflection. This is a misunderstanding of the law of refraction in a continuously varying medium; the same argument, applied to an obliquely fired projectile, would predict that it would never fall but would continue horizontally on reaching its greatest height. The mistake led him to think the mirage could be explained only 
with a wave theory. He constructed such a theory, and it was correct, but rather than being an anticipation of later work, it was a rediscovery of a result published by Airy in 1838.

Raman's collaborator on the theory of the mirage was his talented nephew Pancharatnam, fated soon to die at a tragically early age. Venkataraman gives a detailed account of Pancharatnam's ideas on the interference of polarized light. Only now are we beginning to appreciate their originality and depth - for example his anticipation of the geometric phases now in fashion.

These and many other areas of physics are explained with elegance and clarity. The author is a theoretical physicist with a flair for simple and direct exposition. Sometimes more sophisticated mathematical treatments are merited, and these are given in separate sections so as not to interrupt the main narrative.

Raman seems to have been a difficult and obstinate man. His dealings with administrators and bureaucrats, men of narrow vision compared to him, were stormy, and he made enemies among his colleagues. He became embroiled in controversy and resigned in rage, first from his chair in Calcutta and then from the directorship of the Indian Institute of Science in Bangalore. Finally he founded his own Raman Research Institute in Bangalore. That Institute continues today as a jewel in the crown of Indian science, a tribute to Raman and his successor Radhakrishnan. Venkataraman discusses these matters in great detail and with sensitivity. His book makes us appreciate how unusual Raman must have been to produce such an abundance of creative science, first in the stifling colonial atmosphere of British India, and then in the turbulent struggle for independence.

Michael Berry is a Professor in the H.H. Wills Physics Laboratory, University of Bristol, Tyndall Avenue, Bristol BS8 1TL, UK.

- Oxford University Press will also be the distributor of Raman's collected works, in six volumes and under the title Scientific Papers of C.V. Raman. The books will become available in July-August, price $£ 25, \$ 49.95$ per volume.

\section{Well does He?}

\section{Robert M. May}

Does God Play Dice? The Mathematics of Chaos. By Ian Stewart. Basil Blackwell: 1989. Pp.317. £15. To be published in the United States in May, c. $\$ 19.95$.

READERS OF Nature will recognize Ian Stewart as the person whose News and Views articles continually demonstrate that it is indeed possible to convey the excitement - and the substance - of advances on the frontiers of pure mathematics to a wide audience. Does God Play Dice? is aimed even more widely, to the reading public at large.

The book opens with a far-reaching proposition (with which I agree). In earlier times, the world was seen as unpredictable, governed at best by capricious deities. Over the past few centuries, the newtonian revolution has given us the opposite view, with most things seeming to be predictable provided one has the right rules or equations and enough computing power. But we may be at the start of another swing of the pendulum, with the recognition that many simple and fully deterministic rules give essentially unpredictable or chaotic dynamical behaviour.

In his second and third chapters, Stewart sets the stage with succinct yet lucid accounts of the newtonian paradigm ("Equations for Everything") and of the view that chance or random behaviour derives from complexity ("The Laws of Error"). Poincaré ("The Last Universalist") is seen as bestriding a watershed. Refining the newtonian tradition, he sought qualitative, geometrical ways of understanding the behaviour of systems of differential equations. In the process, he gave birth to the discipline of topology, and initiated the long transition from the classical mechanics of the nineteenth century (with its increasingly elegant solutions of those subsets of equations that could be solved elegantly) to the dynamical systems theory of today. As translated by Stewart, Poincaré recognized what are now called strange attractors in a simplified version of the three-body problem:

When one tries to depict the figure formed by these two curves and their infinity of intersections [one gets] a kind of net, web, or infinitely tight mesh; neither of the two curves can ever cross itself, but must fold back on itself in a very complex way in order to cross the links of the web infinitely many times. One is struck with the complexity of this figure that I am not even attempting to draw.

Stewart launches from this springboard into a lively account of contemporary work on chaos: Smale's formal analysis of strange attractors; the chaotic behaviour (and beautiful regularities) of the quadratic map and other first-order difference equations with 'one hump'; Lorenz's already-classic set of three differential equations as a metaphor for unpredictability in weather systems; the Voyager data and Wisdom's computations on the chaotic tumbling of Hyperion, a moon of Saturn; and much else.

Fractals, the Mandelbrot set and other fashionable topics are also covered, but are clearly tied to the central theme enunciated in the opening chapter. In particular, Stewart gives an excellent, intuitive account of the relation between fractals and chaos (it is not widely appreciated that the geometrical distinction between smooth forms, such as circles and spheres, and rough forms, such as fractal objects, is formally akin to the distinction between the familiar attractors of classical dynamics and the strange attractors of chaos; a fractal dimension can be associated with any given strange attractor, characterizing qualitative features of the dynamics).

Stewart's book will inevitably be compared with James Gleick's deservedly successful Chaos: Making a New Science (Viking, 1987). Both books do a good job of presenting complicated material in a way that is engaging, accurate and accessible to the uninitiated. Both also convey the fascination of the subject. Gleick spends more time on personalities, and on the excitement of the chase. This, I think, may make his account a better read for the layperson, and means that it captures the existential, higgledy-piggledy way science really advances. Gleick's understandable affection for colourful personalities sometimes leads to discrepancies between his 'programme credits' and the perceptions of those in the discipline about who did what first, which has led to some rough reviews by professionals (for example that in Nature 330, 293; 1987).

Although racily written, with lots of staccato paragraphs such as "Simple. Elegant. Elusive." or just "No.", Stewart's book concentrates rather less on people and more on the science itself. Even so, its programme credits are not impeccable. The basic catalogue of the various periodic orbits for generically quadratic maps is attributed to Sharkovskii, whereas among the many independent discoverers I believe the neglected Myrberg comes first; the bifurcation diagram for this map was first published, and its properties of selfsimilarity on smaller and smaller scales noted, by Oster and myself in American Naturalist (110, 573-599; 1976), but here the diagram is as usual called the Feigenbaum tree, permitting a multilingual 'figtree' pun. Such attributions of priority matter only to the players. The play itself is what really counts and here Stewart is, not surprisingly, stronger than Gleick on the mathematics, particularly on how the various aspects of the subject fit together.

In a thought-provoking final chapter Stewart speculates upon the implications of our still-emerging understanding of chaos. Observing that "order can breed its own kind of chaos", he returns to the celebrated exchange between Einstein and Born, and suggests "The question is not so much whether God plays dice, but how God plays dice".

Robert M. May is a Royal Society Research Professor in the Department of Zoology, University of Oxford, South Parks Road, Oxford OX1 3PU, UK, and at Imperial College, London. 